\title{
Relation for the nonequilibrium population of the interface states: Effects on the bias dependence of the ideality factor
}

\author{
G. Gomilaa) and J. M. Rubí \\ Departament de Física Fonamental, Facultat de Física, Universitat de Barcelona, Diagonal 647, \\ 08028 Barcelona, Spain
}

(Received23 August 1996; acceptedfor publication 2 December 1996)

\begin{abstract}
By an analysis of the exchange of carriers through a semiconductor junction, a general relationship for the nonequilibrium population of the interface states in Schottky barrier diodes has been derived. Based on this relationship, an analytical expression for the ideality factor valid in the whole range of applied bias has been given. This quantity exhibits two different behaviours depending on the value of the applied bias with respect to a critical voltage. This voltage, which depends on the properties of the interfacial layer, constitutes a new parameter to complete the characterization of these junctions. A simple interpretation of the different behaviours of the ideality factor has been given in terms of the nonequilibrium charging properties of interface states, which in turn explains why apparently different approaches have given rise to similar results. Finally, the relevance of our results has been considered on the determination of the density of interface states from nonideal current-voltage characteristics and in the evaluation of the effects of the interfacial layer thickness in metal-insulator-semiconductor tunnelling diodes. (C) 1997 American Institute of Physics.
\end{abstract}

[S0021-8979(97)06305-6]

\section{INTRODUCTION}

Metal-semiconductor (MS) contacts have been extensively studied in the literature. ${ }^{1-3}$ For low doping concentrations and moderate temperatures, the interfacial layerthermionic- diffusion (IL-TE-D) theories, which emerged as a synthesis of Bardeen's interfacial layer (IL) ${ }^{4}$ Bethe's thermionic emission (TE) ${ }^{5}$ and Schottky's diffusion $(\mathrm{D})^{6}$ theories, have shown to constitute a powerful tool to analyze, and eventually, self-consistently characterize such junctions. ${ }^{7-11}$ Despite their success in predicting some of the most relevant properties of M-S contacts, they still contain some points which are not well understood or which are still under debate. ${ }^{12}$ In most of the cases, the discussion is centered on the way interface states must exchange carriers under nonequilibrium conditions with either the semiconductor or the metal, and correspondingly on how the interface parameters, as for instance the density of interface states, can be determined from the transport properties of the contact, i.e., nonideal current-voltage (I-V) or capacitance-voltage (C-V) characteristics. To this end, basically two different approaches have been proposed in the literature, namely, those which in some way assume the interface states to be totally or partly in equilibrium (depending on the conditions considered) with either the metal or the semiconductor, ${ }^{2,8,13}$ and those for which the interface states are described by means of a surface quasi-Fermi level, whose position and bias dependence is determined from a given kinetic model. ${ }^{11,14}$ In both cases, the expressions for the $\mathrm{I}-\mathrm{V}$ or $\mathrm{C}-\mathrm{V}$ characteristics in terms of the interface parameters have been derived and the corresponding procedures to obtain these parameters, have been proposed. However, due to the fact that in their common special cases both approaches seem to give rise to simi-

${ }^{a)}$ Electronic mail: gabriel@ hermes.ffn.ub.es lar results, ${ }^{12,13}$ and to the use of some restricting approximations, no general agreement has been reached regarding the correct way to describe the non-equilibrium charging behaviour of interface states.

The purpose of this paper is to present the derivation of a general model for the nonequilibrium population of the interface states. This derivation will be based on a complete analysis of the carrier exchange processes through a semiconductor junction. In particular, no restriction on the exchange of carriers between interface states and either the metal or the semiconductor will be assumed. As a consequence, the model will shown to be directly applicable in the whole range of applied bias. This fact will turn out to be quite important when analyzing the effects of interface states on the bias dependence of the ideality factor. Important results regarding the general behaviour of the bias dependent ideality factor and the nonequilibrium charging properties of interface states will be derived. In particular, it will be explained why apparently different approaches have given rise to similar results in a limited range of bias. In order to present a more complete view of the interfacial properties, both the equilibrium and non-equilibrium formulations of the model will be introduced, although here we will mainly focus on the non-equilibrium case.

The organization of the paper is as follows: In Section II, the equilibrium formulation of the model is presented, together with the introduction of some quantities of interest. The generalization of the model to nonequilibrium conditions is developed in Section III, where the new relation for the nonequilibrium population of interface states is presented. Section IV is devoted to the derivation of the effects of interface states on the bias dependence of the ideality factor and to its experimental corroboration. Finally, in Section $\mathrm{V}$ we sum up our main results. 


\section{EQUILIBRIUM BARRIER HEIGHT IN IL-TE-D THEORIES}

The equilibrium barrier height of $\mathrm{M}-\mathrm{S}$ junctions is one of the most important parameters used to characterize such junctions. ${ }^{1-3}$ In IL-TE-D theories, one assumes the presence of a thin insulating layer sandwiched in between the metal and the semiconductor, and surface states to be present at the semiconductor surface. Under these circumstances, the equilibrium barrier height, $\phi_{b n}^{0}$, defined as the difference between the semiconductor conducting band edge and the metal quasi-Fermi level (we will neglect in this paper image-force effects), is shown to depend strongly on the properties of the interface itself. ${ }^{7,9,10}$ A simple relationship between the barrier height and the interface properties can be derived as follows. Due to the presence of the insulating layer one has ${ }^{1,9}$

$$
\Delta^{0}=\left(\phi_{m}-\chi\right)-\phi_{b n}^{0},
$$

where $\Delta^{0}$ is the equilibrium potential drop across the insulating layer, $\phi_{m}$ is the metal work function and $\chi$ is the semiconductor affinity. By applying Gauss' theorem one obtains, 1,9

$$
\Delta^{0}=\frac{1}{C_{i}}\left(Q_{s s}^{0}+Q_{s c}^{0}\right),
$$

where $C_{i}=\epsilon_{i} / \delta$, with $\epsilon_{i}$ the permittivity of the insulating layer and $\delta$ its length. Moreover, $Q_{s s}^{0}$ and $Q_{s c}^{0}$ are the net charges accumulated at the surface states and in the semiconductor, respectively. By integrating Poisson's equation one arrives at the following expression for $Q_{s c}^{0},{ }^{1}$

$$
\begin{aligned}
Q_{s c}^{0}= & \left(2 q \epsilon N_{D}\right)^{1 / 2}\left[\left(\phi_{b n}^{0}-V_{n}-\frac{k T}{q}\right)\right. \\
& \left.+\frac{k T}{q} e^{-\frac{q}{k T}\left(\phi_{b n}^{0}-V_{n}\right)}\right]^{1 / 2},
\end{aligned}
$$

where $\epsilon$ is the semiconductor permittivity, $N_{D}$ the number density of donors, $q$ the electron charge, $k$ the Boltzmann constant, $T$ the temperature and $V_{n}=(k T / q) \ln \left(N_{C} / N_{D}\right)$, with $N_{C}$ the effective density of states in the conduction band. Usually, the exponential term in Eq. (3) is neglected. Finally, the net surface charge may be evaluated as

$$
Q_{s s}^{0}=-q\left[n_{s s}\left(E_{F s}-E_{V}^{0}\right)-N_{D s}\right],
$$

where $n_{s s}=n_{s s}\left(E_{F s}-E_{V}^{0}\right)$ is a sort of surface equation of state, which is a function of the position of the surface Fermi level $E_{F s}$ relative to the top of the semiconductor valence band, $E_{V}^{0}$. It follows, for instance, from the relation ${ }^{8}$

$$
n_{s s}=\int_{E_{V}^{0}}^{E_{C}^{0}} D_{s}(E) f_{s}(E) \mathrm{d} E,
$$

where $D_{s}(E)$ is the total density of surface states and $f_{s}(E)$ is the occupation function of surface states. We will assume $f_{s}(E)$ to be given through the Fermi-Dirac (FD) distribution

$$
f_{s}(E)=\frac{1}{1+e^{(1 / k T)\left(E-E_{F s}\right)}},
$$

where the Fermi level for the surface states, $E_{F s}$, is used. Furthermore, in Eq. (4) $N_{D s}$ is given by ${ }^{8}$

$$
N_{D s}=\int_{E_{V}^{0}}^{E_{C}^{0}} D_{s}^{d}(E) d E,
$$

with $D_{s}^{d}(E)$ being the density of donor type surface states. Under equilibrium conditions, and provided the semiconductor-insulator interface is abrupt, the surface and semiconductor Fermi levels coincide, that is, $E_{F s}=E_{F n}$. In that case we may relate the surface Fermi level to the equilibrium barrier height as follows:

$$
E_{F s}-E_{V}^{0}=-q \phi_{b n}^{0}+\left(E_{C}^{0}-E_{V}^{0}\right),
$$

where $E_{C}^{0}-E_{V}^{0}$ is the semiconductor band gap. It should be noted that, in the present formulation, $N_{D s}$ and $E_{C}^{0}-E_{V}^{0}$ appear, in principle, as two independent parameters. Only under special circumstances they can be gathered into a single parameter, as for instance, in the case in which one assumes that the density of surface states, $D_{s}$, is a constant through the semiconductor band gap and that the zero temperature occupation function applies. In this situation, the surface equation of state is given by $n_{s s}=D_{s}\left(E_{F s}-E_{V}^{0}\right)$. Then, by defining a neutral level $\phi_{0}$ through the relation $N_{D s}=D_{s} q \phi_{0}$, and by using Eqs. (4) and (8), one arrives at $Q_{s s}^{0}=-q D_{s}\left[-q \phi_{b n}^{0}-\left(q \phi_{0}+E_{V}^{0}-E_{C}^{0}\right)\right]$ where indeed only one parameter, $q \phi_{0}-E_{g}$, appears. ${ }^{7}$ Eqs. (1) - (8) completely determine $\phi_{b n}^{0}$ in terms of the surface parameters, giving rise to

$$
\begin{aligned}
\left(\phi_{m}-\chi\right)- & \phi_{b n}^{0} \\
= & -\frac{q}{C_{i}}\left[n_{s s}\left(-e \phi_{b n}^{0}+E_{C}^{0}-E_{V}^{0}\right)-N_{D s}\right]+\frac{\left(2 q \epsilon N_{D}\right)^{1 / 2}}{C_{i}} \\
& \times\left(\phi_{b n}^{0}-V_{n}-\frac{k T}{q}+\frac{k T}{q} e^{-\frac{k T}{q}\left(\phi_{b n}^{0}-V_{n}\right)}\right)^{1 / 2} .
\end{aligned}
$$

Particular cases of this relation have been analyzed elsewhere. ${ }^{7,9,10}$ In this respect, it is worth noting that, even in the case that the second term on the ride hand side is negligible, the relationship between the metal work function and the barrier height does not need to be linear due to the possible nonlinearity associated with the dependence of $n_{s s}$ on the barrier height. This is an important point with respect to the internal consistency of the model, because nonconstant densities of interface states, which correspond to nonlinear surface equations of state, are commonly assumed in order to interpret the transport properties of these junctions, and hence they should also be used to interpret their equilibrium properties.

\section{THE NONEQUILIBRIUM MODEL}

In the nonequilibrium version of the IL-TE-D theories two current limiting mechanisms are assumed to control the transport properties of the junction, namely, the diffusion of carriers through the semiconductor and the exchange of carriers through the junction. As usual, we will describe the diffusion of carriers through the semiconductor by means of a drift-diffusion like relation ${ }^{1,15}$ 


$$
J_{n}(x)=-D_{n} \frac{\partial n(x)}{\partial x}-\mu_{n} n(x) E(x),
$$

where $J_{n}$ is the electron number density current, $n(x)$ the electron number density, $E$ the electric field and $D_{n}$ and $\mu_{n}$, the diffusion and mobility coefficients. With respect to the exchange of carriers through the junction we will assume here a kinetic approach in which the exchange of carriers takes place via three different mechanisms ${ }^{16}$ (for simplicity, in this paper we neglect the effects of minority carriers, although the generalization to include them is straightforward), namely, the exchange of carriers, via tunnelling, between the metal and either the interface states or the semiconductor and the exchange of carriers, with no tunnelling, between the interface states and the semiconductor. These three processes can be identified by means of the following elementary kinetic processes

$$
q_{n} \rightleftharpoons q_{m} ; \quad q_{s} \rightleftharpoons q_{m} ; \quad q_{s} \rightleftharpoons q_{n},
$$

where $q_{m}, q_{n}, q_{s}$ stands for the electrons in the metal, in the semiconductor, and at the interface states, respectively. It is worth pointing out, that these three processes constitute a coupled set of kinetic processes and as such, they must be treated as a whole. This fact has not been taken into account in previous analyses.

With the purpose of analyzing the global dynamics corresponding to this set of coupled kinetic processes we will make use of the Shockley-Read-Hall (SRH) statistics ${ }^{17,18}$ (see Appendix), although equivalent results may be obtained by other methods. ${ }^{16}$ Under stationary conditions, this treatment states that the net exchange of carriers between metal and semiconductor is given by the following thermionic emission-like relation (see Appendix)

$$
J_{n}=N_{C} V_{R} e^{-(q / k T) \phi_{b n}}\left(1-e^{(1 / k T)\left(F_{n}-F_{m}\right)}\right),
$$

and that the nonequilibrium position of the surface quasiFermi level is given by (see Appendix)

$$
e^{(1 / k T)\left(F_{s}-F_{m}\right)}=\frac{1+\alpha^{-1} e^{(1 / k T)\left(F_{n}-F_{m}\right)}}{1+\alpha^{-1}},
$$

where $F_{n}-F_{m}\left(F_{s}-F_{m}\right)$ stands for the jump in the electron quasi-Fermi level between the metal and the semiconductor surfaces (interface states and the metal surface). Note that due to the coupling between the kinetic processes both results have been derived in a unified way, and not by introducing different assumptions to obtain each one. Furthermore, in Eqs. (12) and (13), $\phi_{b n}=E_{C}-F_{m}$ is the nonequilibrium barrier height and $V_{R}$ and $\alpha$ are two positive quantities given through (see Appendix and Ref. 16)

$$
\begin{aligned}
& N_{C} V_{R}=\lambda_{n m}+\frac{e^{(1 / k T)\left(E_{C}^{0}-E_{V}^{0}\right)}}{\left(\lambda_{s n}\right)^{-1}+\left(\lambda_{s m}\right)^{-1}}, \\
& \alpha=\frac{\lambda_{s m}}{\lambda_{s n}},
\end{aligned}
$$

where $\lambda_{n m}, \lambda_{s m}, \lambda_{s n}$, stands for the transition coefficients corresponding to the elementary processes represented in Eq. (11). Explicit expressions for these coefficients are also given in the Appendix.
To take into account the fact that the kinetic processes between the metal and either the interface states or the semiconductor take place via tunnelling, we will assume following Refs. 15 and 19, that, as a first approximation, the corresponding transmission coefficients, $\lambda_{s m}$ and $\lambda_{n m}$, can be expressed as

$$
\lambda_{n m}=\bar{\lambda}_{n m} \theta_{n m}, \lambda_{s m}=\bar{\lambda}_{s m} \theta_{s m},
$$

where $\bar{\lambda}_{n m}$ are the values corresponding to abrupt junctions (but with a discontinuous potential, see Appendix) and

$$
\theta_{n m}=e^{-\phi_{n m}^{1 / 2} \delta}, \quad \theta_{s m}=e^{-\phi_{s m}^{1 / 2} \delta}
$$

are the transmission coefficients of the insulating layer, with $\phi_{n m}, \phi_{s m}$ being related to the corresponding effective barrier height presented by the insulating layer to the tunnelling of carriers. ${ }^{15,19}$ Here $\delta$ refers to the length of the insulating layer. This simple dependence on the interfacial layer thickness, qualitatively incorporates the major features of the tunnelling of carriers through the insulating layer, because for abrupt junctions, $(\delta=0), \theta_{n m}$ and $\theta_{s m}$ are equal to one, and we do not have any tunnelling effect, while for thick insulating layers ( $\delta$ large), $\theta_{n m}$ and $\theta_{s m}$ vanish quickly, thus inhibiting the exchange of carriers with the metal, as should be.

At this point, some comments are in order. First of all, it should be noted that Eq. (12) is formally identical to the commonly used TE relation ${ }^{1,10,15}$ but here it appears with a modified $V_{R}$ factor that incorporates the effects of the interface states, Eq. (14). As mentioned above, this result is reminiscent of the fact that the underlying kinetic processes are not independent. Furthermore, Eq. (13), which is one of the main results of the present paper, constitutes a new general expression governing the nonequilibrium position of the surface quasi-Fermi level, and hence the nonequilibrium population of the interface states. As will be seen, it allows a complete description of the exchange processes through MS contacts. Note that both the insulating layer thickness and the applied bias, implicitly present in $\alpha$ and $F_{n}-F_{m}$, respectively, contribute to determine this position.

In order to compare our result with previously existing ones, it should be emphasized that our model has been formulated for the general case of a continuous distribution of surface states, $D_{s}(E)$, through the semiconductor band gap. Depending on the precise form of this distribution, different expressions for the $\lambda$ 's, and hence for $V_{R}$ and $\alpha$, and for the surface equation of state, $n_{s s}$, can be obtained. By taking these facts into account, it is easily shown that two of the expressions reported in Ref. 14, constitute two particular cases of our general result, Eq. (13). However, the fact that they neglect the discontinuity in the electric potential through the insulating layer, limits considerably the application of their model, while in our model this limitation does not appear. Moreover, those models in which the interface states are assumed to be in equilibrium for all bias values with the metal, $F_{s}=F_{m}$, or with the semiconductor, $F_{s}=F_{n}$ can be seen to correspond to either tending $\alpha^{-1}$ or $\alpha$ to zero in Eq. (13), respectively. The case in which these models applies for a limited range of bias values will be discussed at the end of Section IV. Finally, the case in which part of the interface states are in equilibrium with the metal 
and part with the semiconductor, ${ }^{19}$ will be shown to also be recoverable from our result [Eq. (13)] in a generalized way (see Section IV), by simply assuming a finite value for $\alpha$. These comparisons with previously reported results just show the great generality and wide applicability of our model, and hence its ability for giving a unified view of the transport properties of MS contacts.

In order to render our model complete we need to formulate the corresponding nonequilibrium versions of Eqs. (1), (2), (4), (8). We obtain

$$
\begin{aligned}
& \Delta=\left(\phi_{m}-\chi\right)-\phi_{b n}, \\
& \Delta=\frac{1}{C_{i}}\left(Q_{s s}+Q_{s c}\right), \\
& Q_{s s}=-q\left[n_{s s}\left(F_{s}-E_{V}\right)-N_{D s}\right], \\
& F_{s}=E_{V}+\left(F_{s}-F_{m}\right)-q \phi_{b n}+E_{C}^{0}-E_{V}^{0},
\end{aligned}
$$

where in Eq. (19) we have neglected a contribution proportional to the bulk electric field and in Eq. (21) $\left(F_{s}-F_{m}\right)$ is given through Eq. (13). Finally, $Q_{s c}$ in Eq. (19) may be computed by integrating Poisson's equation together with Eq. (10). In this respect, it should be remembered that in the IL-TE-D models the total applied bias splits up into two contributions

$$
q V=\left(F_{n}-F_{m}\right)+q V_{b},
$$

where $q V_{b}=F_{n}(w)-F_{n}(0)$ is the drop in applied bias across the active region of the bulk semiconductor, whose length is $w$, and $F_{n}-F_{m}$ the corresponding drop across the junction. These remarks end the general formulation of the model.

\section{EFFECTS OF INTERFACE STATES ON THE BIAS DEPENDENCE OF THE IDEALITY FACTOR}

As a first application of our model we will analyze in this section the effects of interface states on the bias dependence of the ideality factor. The direct applicability of our model to the whole range of bias will allow us to draw important conclusions regarding the nonequilibrium population of the interface states and, in particular, to justify why different approaches have given rise to similar results in a limited range of bias. To the end of obtaining analytical results, the calculations will be carried out for the case in which the thermionic approximation holds, ${ }^{2}$ although the general case can also be analyzed.

As a first step in deriving the bias dependence of the ideality factor, we need to find the expression for the I-V characteristics. As is well-known, for IL-TE-D theories it may be obtained from Eqs. (10) and (12), and is given by ${ }^{15}$

$$
\left.I=q N_{C} V_{R D} e^{-(q / k T) \phi_{b n}\left[e^{(q / k T)} V_{-}\right.} 1\right],
$$

where we have introduced the density of electric current $I=-q J_{n}$, and defined $V_{R D}=V_{R} /\left[1+\left(V_{R} / V_{D}\right)\right]$ with

$$
V_{D}=\frac{D_{n}}{\int_{0}^{w} e^{-(q / k T)[V(x)-V(0)]} d x} .
$$

For practical purposes, we will rewrite Eq. (23) as
$I=q N_{C} V_{R D}^{0} e^{-(q / k T) \phi_{b n}^{0}} e^{-(q / k T)\left(\Delta \phi_{b n}+\Delta \phi_{b}^{R D}\right)}\left[e^{(q / k T) V}-1\right]$,

where the quantities with a superscript 0 refer to the equilibrium value. Moreover, $\Delta \phi_{b n}=\phi_{b n}-\phi_{b n}^{0}$ and $\Delta \phi_{b}^{R D}$ $=(k T / q) \ln \left(V_{R D}^{0} / V_{R D}\right)$. Eq. (25), or alternatively Eq. (23), constitutes the formal expression for the I-V characteristics in IL-TE-D theories, ${ }^{15}$ where $\Delta \phi_{b n}$ and $\Delta \phi_{b}^{R D}$ are two biasdependent quantities to be determined. As mentioned previously, the thermionic emission approximation enables us to arrive at explicit expressions for them. Indeed, under this approximation, in which one assumes ${ }^{2} V_{R} / V_{D} \ll 1$, one has $\Delta \phi_{b}^{R D} \approx 0$ (see its definition above), and hence the nonideality in the I-V curve is only due to the bias dependence of $\Delta \phi_{b n}$. In deriving this result it has been implicitly assumed that $V_{R}$ does not depend significantly on bias. Moreover, from Eqs. (10) and (13), one arrives at ${ }^{10}$

$$
e^{(1 / k T)\left(F_{n}-F_{m}\right)}=\frac{1+\frac{V_{D}}{V_{R}} e^{(q / k T) V}}{1+\frac{V_{D}}{V_{R}}},
$$

which shows that in this limit one may approximate $\left(F_{n}-F_{m}\right) \approx V$, which is equivalent to assume a flat quasiFermi level through the active region of the contact. This last result considerably simplifies the calculations, firstly because the bias dependence of $F_{s}-F_{m}$ is now given directly through Eq. (13),

$$
e^{(1 / k T)\left(F_{s}-F_{m}\right)}=\frac{1+\alpha^{-1} e^{(q / k T) V}}{1+\alpha^{-1}},
$$

and second, because $Q_{s c}$ may be explicitly evaluated as ${ }^{1,2}$

$$
Q_{s c}=q N_{D} w,
$$

with

$$
w=\left(\frac{2 \epsilon}{q N_{D}}\right)^{1 / 2}\left(\phi_{b n}-V-V_{n}-\frac{k T}{q}\right)^{1 / 2},
$$

where in the last expression an exponential term similar to the one appearing in Eq. (3) has been neglected. By taking into account Eqs. (18)-(21), (28) and (29), we obtain the following expression involving $\phi_{b n}$,

$$
\begin{aligned}
\left(\phi_{m}-\chi\right)-\phi_{b n}= & -\frac{q}{C_{i}}\left\{n _ { s s } \left[\left(F_{s}-F_{m}\right)-q \phi_{b n}+E_{C}^{0}\right.\right. \\
& \left.\left.-E_{V}^{0}\right]-N_{D s}\right\}+\left(\frac{2 q \epsilon N_{D}}{C_{i}}\right)^{1 / 2} \\
& \times\left(\phi_{b n}-V-V_{n}-\frac{k T}{q}\right)^{1 / 2},
\end{aligned}
$$

where no particular surface equation of state has been specified. Apart from this fact, the main difference between our result and previously reported ones comes from the bias dependence of the surface quasi-Fermi level position, represented by $\left(F_{s}-F_{m}\right)$. Here, we have shown it to be given through Eq. (27) in this approximation. Eq. (30) gives the complete bias dependence of the barrier height and therefore it completely determines the I-V characteristics. 
Now we are in a position to derive the bias dependence of the ideality factor in the whole range of biases. To this end, we will use the general definition of the ideality factor, ${ }^{2}$

$$
\frac{1}{n}=1+\frac{k T}{q} \frac{d}{d V} \ln \left(\frac{I}{e^{(q / k T) V}-1}\right),
$$

which holds for all values of bias (forward, reverse, and the transition between both). By means of this definition and using the thermionic emission approximation we have [see Eq. (23)],

$$
\frac{1}{n}=1-\frac{d \phi_{b n}}{d V}
$$

which, by taking into account Eq. (30), allows us to derive the following analytical expression for $n$,

$$
n=1+\frac{\frac{1}{C_{i}}\left(\frac{\epsilon}{w}+q^{2} D_{s b}\right)}{1+\frac{q^{2} D_{s a}}{C_{i}}},
$$

where we have defined $D_{s b}$ and $D_{s a}$ as

$$
D_{s a}=D_{s}\left(1-\frac{1}{q} \frac{d\left(F_{s}-F_{m}\right)}{d V}\right), D_{s b}=D_{s} \frac{1}{q} \frac{d\left(F_{s}-F_{m}\right)}{d V},
$$

with

$$
D_{s}=\left.\frac{\partial n_{s s}}{\partial E_{F s}}\right|_{E_{F s}-E_{V}^{0}=\left(F_{s}-F_{m}\right)-q \phi_{b n}+E_{C}^{0}-E_{V}^{0}}
$$

being the apparent density of surface states, ${ }^{8}$ and where [see Eq. (27)]

$$
\frac{1}{q} \frac{d\left(F_{s}-F_{m}\right)}{d V}=\frac{1}{1+e^{-(q / k T)\left(V-V_{c}\right)}}
$$

with $V_{c}=(k T / q) \ln \alpha$. Note that in performing this last derivative we have assumed $\alpha$ to be approximately bias independent. Eq. (33), together with Eqs. (34) and (35) which give the bias dependence of $D_{s b}$ and $D_{s a}$, completely determine the bias dependence of the ideality factor and constitutes an expression directly applicable to the whole range of bias values without the need of additional assumptions. It is worth noting that despite the fact that we have used a kinetic approach to describe the nonequilibrium charging behaviour of the interface states, a formally equivalent expression for the ideality factor to one used in other approaches, ${ }^{2}$ has been obtained. This fact shows that if under this second approach the appropriate bias dependence for $D_{s b}$ and $D_{s a}$ is assumed, equivalent results may be obtained. Note that under our approach this dependence is directly obtained through Eqs. (34) and (35).

In Fig. 1 we have represented the bias dependence of the ideality factor given through Eq. (33), for the case of a constant density of surface states, $D_{s}$. Different values of the critical voltage, $V_{c}$, and different situations of interest, (a) $(\epsilon / w) / C_{i} \ll 1$ and (b) $(\epsilon / w) / C_{i} \approx 1$, have been considered. From these figures it is clearly concluded, that the ideality factor displays two remarkably different behaviours depend-
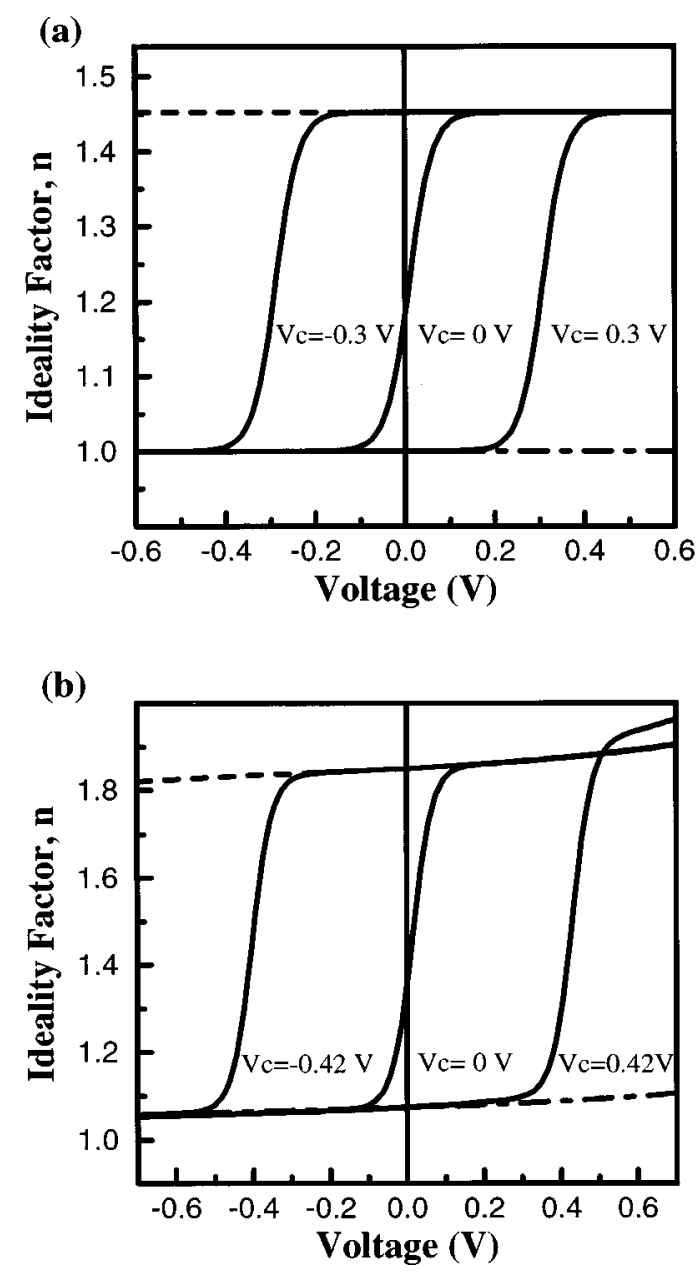

FIG. 1. Ideality factor as a function of bias, for different values of the critical voltage, $V_{c}$ (continuous line). The density of interface states, $D_{s}$, has been assumed constant. (a) $D_{s}=2.5 \times 10^{14} \mathrm{eV}^{-1} \mathrm{~cm}^{-2}, C_{i}=8.85$ $\times 10^{-5} \mathrm{~F} / \mathrm{cm}^{2}, N_{D}=10^{15} \mathrm{~cm}^{-3}$, and (b) $D_{s}=4 \times 10^{12} \mathrm{eV}^{-1} \mathrm{~cm}^{-2}, C_{i}$ $=8.85 \times 10^{-7} \mathrm{~F} / \mathrm{cm}^{2}, N_{D}=10^{17} \mathrm{~cm}^{-3}$. Nonspecified parameters correspond to GaAs (see Ref. 1). Also plotted for $V_{c}=-0.3 \mathrm{~V}$ and $V_{c}=$ $-0.42 \mathrm{~V}$ (b) the two approximations (dashed and dotted-dashed lines).

ing on whether the bias voltage is larger or smaller than the critical voltage, $V_{c}$. As this voltage depends on the properties of the interfacial layer, it constitutes a relevant parameter in the characterization of MS contacts. We note, for instance, that depending on the sign of $V_{c}$, the transition between both behaviours takes place under forward $\left(V_{c}>0\right)$ or reverse bias $\left(V_{c}<0\right)$. In practice, negative or around zero values of $V_{c}$ are expected to occur, corresponding to MIS tunneling diodes (large values of $\delta$ ), and to Schottky barriers diodes (small values of $\delta$ ), respectively [see Eqs. (15), (16), (17) and the definition of $V_{c}$ in terms of $\alpha$ given after Eq.(35)]. In Fig. 2 the ideality factor as a function of bias have been plotted for different values of $D_{s}$ and for a given value of $V_{c}$, for the case that $(\epsilon / w) / C_{i} \ll 1$. Note that independent of the value of $D_{s}$, the ideality factor always become very close to unity for low enough values of bias. Furthermore, in all cases this fact seems to happen at about the same value of the applied bias, $V_{0}$, which is related to $V_{c}$ by $V_{0} \approx V_{c}$ $-3(k T / q)$. This result constitutes a very useful result in practice, because it allows us to identify the critical voltage 


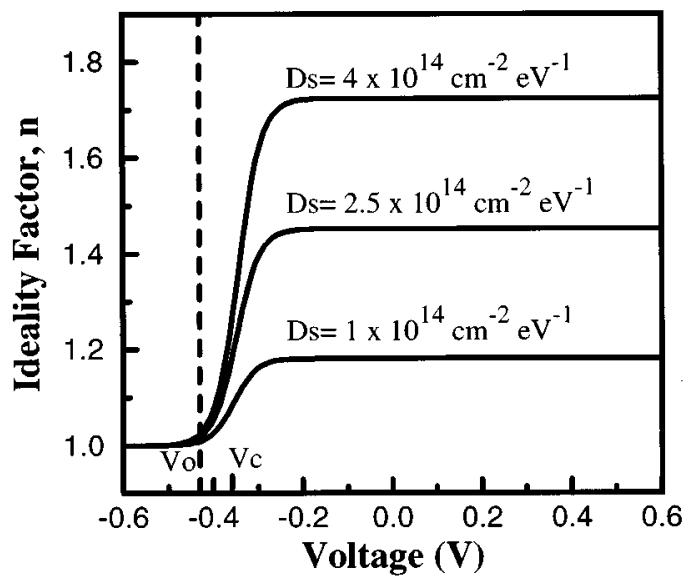

FIG. 2. Ideality factor vs applied voltage, for different values of the density of interface states, $D_{s}$. The critical voltage is equal to $-0.36 \mathrm{~V}$. Same parameters as in Fig. 1(a). The dashed line corresponds to $V_{0}=V_{c}-3 k T / q \approx-0.43 \mathrm{~V}$.

$V_{c}$ directly from these type of plots. Indeed, by simply identifying the bias at which $n$ becomes both independent of bias and practically unity, let say $n \approx 1.01$, we may obtain $V_{0}$, from which $V_{c}$ can be approximately determined.

A simple interpretation of the different behaviours presented by the ideality factor may be given in terms of the nonequilibrium charging behaviour of the interface states. By noting that for $V>V_{c}+3(k T / q)$ the derivative in Eq. (35) may be approximated by unity, one has from Eq. (34), $D_{s a} \approx 0$ and $D_{s b} \approx D_{s}$. We then conclude that, for these voltages, interface states behave as if they all were in equilibrium with the semiconductor. As long as this approximation holds, the ideality factor is simply given by

$$
n \approx 1+\frac{1}{C_{i}}\left(\frac{\epsilon}{w}+q^{2} D_{s}\right), \quad V>V_{c}+3 \frac{k T}{q} .
$$

On the other hand for $V<V_{c}-3(k T / q)$ we may approximate the derivative in Eq. (35) by zero and hence $D_{s a} \approx D_{s}$ and $D_{s b} \approx 0$. As a consequence for these bias values, the interface states behave as if they all were in equilibrium with the metal, and the ideality factor is given by

$$
n \approx 1+\frac{1}{C_{i}} \frac{\frac{\epsilon}{w}}{1+\frac{q^{2} D_{s}}{C_{i}}}, \quad V<V_{c}-3 \frac{k T}{q} .
$$

Hence, following our model the two different behaviours displayed by the ideality factor can be associated to different effective equilibrations of the interface states with either the semiconductor $\left[V>V_{c}+3(k T / q)\right]$ or the metal $\left[V>V_{c}-3(k T / q)\right]$, (see Fig. 3).

At this point some remarks are in order. In Fig. 1, we have represented both Eqs. (36) (discontinuous line) and (37) (discontinuous-dotted line) for $V_{c}=-0.3 \mathrm{~V}$ (a) and $V_{c}=$ - $0.42 \mathrm{~V}(\mathrm{~b})$. Excellent agreement with the exact values, given through Eq. (33), is observed, in the range in which the approximations hold. This fact implies that, in general, it is not true that Eqs. (36) and (37) hold under forward and re-

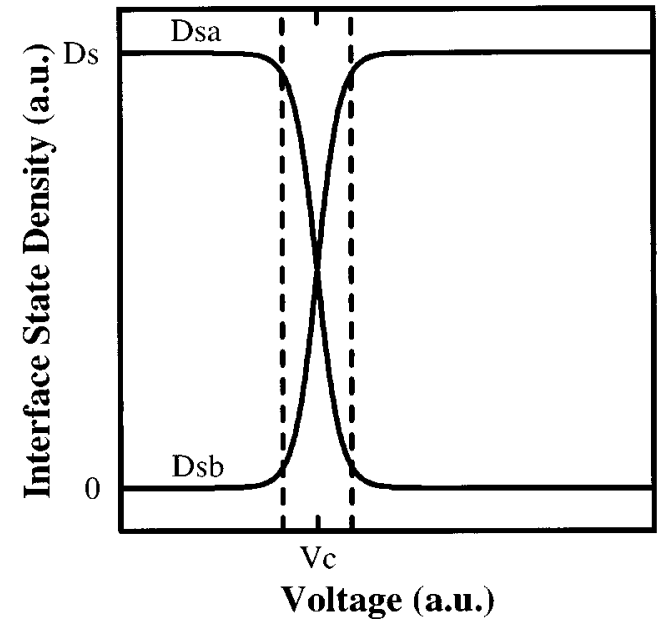

FIG. 3. $D_{s b}$ and $D_{s a}$ as a function of bias, for a constant density of interface state, $D_{s}$. The dashed lines correspond to the voltage values $V_{c}-3 k T / q$ and $V_{c}+3 k T / q$.

verse bias, respectively, as has been sometimes implicitly assumed. As has been shown above, only a precise determination of $V_{c}$ allows us to determine the range of applicability of these approximate relations. As a result, depending on the value of $V_{c}$, the range for which Eq. (36) can be applied to determine $D_{s}$ may turn out to be larger (or smaller) than previously assumed. Furthermore, the value of $V_{c}$ itself may provide some useful information about the effects of the interfacial layer thickness on the transport properties of these junctions, a fact of great practical interest in studying MIS tunneling diodes and some of their applications, as the solar cells.

Some recently reported experimental I-V characteristics $^{20-23}$ have been analyzed in order to corroborate the predictions of our model. The corresponding bias dependence of the ideality has been computed by means of its definition Eq. (31). Regarding the behaviour of the ideality factor, it is interesting to note that in all the cases this quantity behaves in a similar way. Thus, below a certain given voltage, whose value depends on the system considered [for instance, around $-1 \mathrm{~V}$ and $-0.9 \mathrm{~V}$ for samples (a) and (b), respectively, in Ref. $22,-0.25 \mathrm{~V}$ for sample $D_{1}$ in Ref. 20, between $-0.15 \mathrm{~V}$ and $0 \mathrm{~V}$ for samples in Ref. 23 and between $-0.25 \mathrm{~V}$ and $0 \mathrm{~V}$ for samples in Ref. 21], the ideality factor becomes practically independent of the bias and approaches unity. Above these voltages, it becomes bias dependent, presenting sometimes, in a limited range of biases, a plateau corresponding to the reported value of the ideality factor $[n=1.180$ and $n=1.107$ for samples (a) and (b), respectively, in Ref. 22, or $n=1.25$ for sample $D_{1}$ in Ref. 20]. In view of these features we may conclude that these experimental results strongly support the predictions of our model. Indeed, the fact that $n$ becomes in all cases nearly unity below a certain bias implies that for all of them we may assume $(\epsilon / w) / C_{i} \ll 1$ (see Fig. 2). As a consequence, these voltages can be identified with $V_{0}$, from which the values of $V_{c}$ can be roughly determined. Some of the extracted values are: around $-0.9 \mathrm{~V}$ and $-0.8 \mathrm{~V}$ for samples (a) and (b), respectively in Ref. 22 , around $-0.15 \mathrm{~V}$ for sample $D_{1}$ in 
Ref. 20, between $-0.1 \mathrm{~V}$ and $0.1 \mathrm{~V}$ for samples in Ref. 23 and between $-0.2 \mathrm{~V}$ and $0.1 \mathrm{~V}$ for samples in Ref. 21. As is seen, $V_{c}$ depends considerably on the system considered. Furthermore, the large negative values obtained could be explained in terms of the dependence of $V_{c}$ on the $\delta$. Finally, the region in which the experimental values for $n$ become bias dependent, corresponds to the region of bias voltages satisfying $V>V_{c}+3(k T / q)$. There, Eq. (36) holds and, as usual, it can be applied to determine the density of interface states. As mentioned previously, for the case of negative values of $V_{c}$, a considerable large region of biases can be investigated to this end.

It is worth emphasizing that our model provides a very natural and general way of interpreting these experimental results in the whole range of bias voltages. The previously existing models for which the interface states are assumed to be in equilibrium with the metal (semiconductor) can only give correct results when they are applied to bias voltages below (above) $V_{c}$, but not in the whole range of bias. On the other hand, those models which assume that part of the interface states are in equilibrium with the metal and part with the semiconductor ${ }^{19}$ will give correct results in the whole range of bias only if they consider the bias dependence for $D_{s a}$ and $D_{s b}$ derived in this work [see Eq.(34)]. Otherwise, results are restricted to particular cases and are only valid for certain regions of applied bias. This is the case for instance when it is assumed that $D_{s a} \approx 0$ and $D_{s b} \approx D_{s}$, under forward bias, and $D_{s a} \approx D_{s}$ and $D_{s b} \approx 0$, under reverse bias. ${ }^{13}$ In this case, correct results will be obtained only if the system satisfies $V_{c} \approx 0$, and for voltages outside the transition region. Finally, we want to consider also the case in which one assumes that $D_{s a} \approx 0$ and $D_{s b} \approx 0$, under reverse bias conditions, in order to interpret the apparent independence of the results on the population of the interface states. ${ }^{13}$. Following our results, we think that a more satisfactory explanation of this fact may be obtained by assuming that $D_{s a} \approx D_{s}$ and $D_{s b} \approx 0$ below $V_{c}$, which for $V_{c} \approx 0$ coincides with the reverse bias region, but with $(\epsilon / w) / C_{i} \ll 1$. As has been seen shown before under this circumstances similar results are predicted by our model (see Fig. 2).

\section{CONCLUSIONS}

In this paper we have presented a general IL-TE-D theory for Schottky barrier diodes. This theory, which has been based upon rather realistic assumptions about the exchange of carriers through a semiconductor junction, has allowed us to overcome some of the inherent limitations of the previous existing theories and therefore to develop a more complete analysis of the effects of interface states on the transport properties of MS contacts. The main novelty incorporated in our theory, aside from minor changes in the TElike relation or some implications in the evaluation of the equilibrium barrier height, consists of a new general relation to control the nonequilibrium population of the interface states, Eq. (13). By means of this relation, and in the thermionic emission limit, an analytic expression for the ideality factor, as a function of the applied voltage, has been derived, which has been shown to be directly applicable to the whole range of voltages (forward, reverse and the transition be- tween them). The analysis of this relation has allowed us to identify two different behaviours of the ideality factor, depending on whether the applied bias is greater or smaller than a certain critical voltage $V_{c}$, a quantity that constitutes a new parameter to be determined in order to complete the characterization of these contacts. A direct relation between these two behaviours and the nonequilibrium charging properties of the interface states has been established, which in particular has explained why different approaches developed previously have given rise to similar results in a limited range of bias values. Experimental results strongly support our predictions opening then the possibility of a more complete determination of the density of interface states from non-ideal I-V characteristics or of a better evaluation of the interfacial layer thickness effects in MIS tunnelling diodes.

\section{ACKNOWLEDGMENTS}

This work has been supported by DGICYT of the Spanish Government under grant PB92-0859 and the European Union Human Capital and Mobility Programme, contract ERB-CHR-XCT93-0413. One of us (G.G.) wishes to thank CIRIT of Generalitat de Catalunya for financial support.

\section{APPENDIX A: SRH STATISTICS FOR THE EXCHANGE OF CARRIERS THROUGH A JUNCTION}

The purpose of this appendix is to derive Eqs. (12) and (13) by means of the SRH statistics applied to the set of kinetics processes represented in Eq. (11). Given these elementary kinetic processes, the net conducting current at the semiconductor surface, $J_{n}$, and the net current from the interface states, $J_{s}$, are given by

$$
\begin{aligned}
& J_{n}=J_{n m}+J_{n s}, \\
& J_{s}=J_{n s}+J_{m s},
\end{aligned}
$$

where $J_{a b}$ (with $a, b=n, m, s$ ) are the kinetic rates corresponding to the elementary process $q_{a} \rightleftharpoons q_{b}$. By applying the SRH statistics ${ }^{17,18}$ to these elementary processes, the following expressions for the rates can be derived, ${ }^{24}$

$$
\begin{aligned}
J_{a b}= & \int d E_{a} \int d E_{b} D_{a}\left(E_{a}\right) D_{b}\left(E_{b}\right)\left\{f_{a}\left(E_{a}\right)\left[1-f_{b}\left(E_{b}\right)\right]\right. \\
& \left.\times \gamma_{a b}\left(E_{a}, E_{b}\right)-f_{b}\left(E_{b}\right)\left[1-f_{a}\left(E_{a}\right)\right] \gamma_{b a}\left(E_{a}, E_{b}\right)\right\},
\end{aligned}
$$

where $D_{a}\left(E_{a}\right)$ is the density of states of system $a, f_{a}\left(E_{a}\right)$ its occupation function, given through the FD distribution $f_{a}\left(E_{a}\right)=\left(1+e^{\beta\left(E_{a}-E_{F a}\right)}\right)^{-1}$, with $E_{F a}$ the corresponding Fermi level, and $\gamma_{a b}\left(E_{a}, E_{b}\right)$ is the probability per unit time for the transition between states of energy $E_{a}$ and $E_{b}$. In order for the square bracket to be dependent on only the quasi-Fermi level difference, as is usually required, the following generalized detailed balance will be assumed $\gamma_{b a}\left(E_{a}, E_{b}\right)=\gamma_{a b}\left(E_{a}, E_{b}\right) e^{\beta\left(E_{b}-E_{a}\right)} e^{q \beta\left(V_{a}-V_{b}\right)}$. Note that for the abrupt case in which the electric potential is continuous, this relation is equivalent to the usual one ${ }^{17}$ By taking into account this relation, Eq. (A3), can be written as 


$$
\begin{aligned}
J_{a b}= & \int d E_{a} \int d E_{b} D_{a}\left(E_{a}\right) f_{a}\left(E_{a}\right) D_{b}\left(E_{b}\right) \\
& \times\left[1-f_{b}\left(E_{b}\right)\right] \gamma_{a b}\left(E_{a}, E_{b}\right)\left(1-e^{\beta\left(F_{b}-F_{a}\right)}\right),
\end{aligned}
$$

where, indeed, the result is only dependent on the quasiFermi level difference, here defined as $F_{a}=E_{F a}-q V_{a}$. For our purposes, Eq. (A4) will be rewritten as follows

$$
J_{a b}=\lambda_{b a} e^{-\beta\left(E_{R b}-F_{a}\right)}\left(1-e^{\beta\left(F_{b}-F_{a}\right)}\right),
$$

where $E_{R b}=E_{R b}^{0}-q V_{b}$, with $E_{R n}=E_{C}, E_{R S}=E_{V}$ and $E_{R m}=E_{m}$, and

$$
\begin{aligned}
\lambda_{b a}= & \int d E_{a} \int d E_{b} D_{a}\left(E_{a}\right) D_{b}\left(E_{b}\right)\left[1-f_{a}\left(E_{a}\right)\right] \\
& \times\left[1-f_{b}\left(E_{b}\right)\right] \widetilde{\gamma}_{b a}\left(E_{a}, E_{b}\right),
\end{aligned}
$$

where we have defined $\tilde{\gamma}_{b a}\left(E_{a}, E_{b}\right)=\gamma_{b a}\left(E_{a}, E_{b}\right)$ $\times e^{\beta\left(E_{R b}^{0}-E_{b}\right)}$. With these definitions the inverse transition coefficient $\lambda_{a b}$ can be defined from Eq. (A6), by simply interchanging $a$ by $b$ everywhere, and it is easily shown that they satisfy the following detailed balance relation, $\lambda_{a b}$ $=e^{\beta\left(E_{R a}-E_{R b}\right)} \lambda_{b a}$. This generalized detailed balance relation, valid for both nonabrupt systems and nonequilibrium conditions, is totally equivalent to the one derived in Ref. 16 by using a pure phenomenological approach, a fact that strongly supports the assumptions made above.

By substituting the corresponding rates of the form given by Eq. (A5) into Eqs. (A1) and (A2) we obtain

$$
\begin{aligned}
J_{n}= & \lambda_{n m} e^{-\beta\left(E_{C}-F_{m}\right)}\left(1-e^{\beta\left(F_{n}-F_{m}\right)}\right) \\
& +\lambda_{n s} e^{-\beta\left(E_{C}-F_{s}\right)}\left(1-e^{\beta\left(F_{n}-F_{s}\right)}\right), \\
J_{s}= & \lambda_{n s} e^{-\beta\left(E_{C}-F_{s}\right)}\left(1-e^{\beta\left(F_{n}-F_{s}\right)}\right) \\
& +\lambda_{m s} e^{-\beta\left(E_{m}-F_{s}\right)}\left(1-e^{\beta\left(F_{m}-F_{s}\right)}\right),
\end{aligned}
$$

which describe the exchange processes under general conditions. Again, these relations are formally equivalent to the ones derived in Ref. 16. Under stationary conditions, and provided that the effects of minority carriers are neglected, one has $J_{s}=0$. From Eq. (A8) we then obtain

$$
e^{\beta\left(F_{s}-F_{m}\right)}=\frac{1+\left(\frac{\lambda_{s n}}{\lambda_{s m}}\right) e^{\beta\left(F_{n}-F_{m}\right)}}{1+\frac{\lambda_{s n}}{\lambda_{s m}}},
$$

where the detailed balance relation for the $\lambda$ 's has been used. Substituting this expression into Eq. (A7) we finally arrive at

$$
J_{n}=\left(\lambda_{n m}+\frac{e^{\beta\left(E_{C}-E_{V}\right)}}{\lambda_{s n}^{-1}+\lambda_{s m}^{-1}}\right) e^{-\beta\left(E_{C}-F_{m}\right)}\left(1-e^{\beta\left(F_{n}-F_{m}\right)}\right),
$$

where again the detailed balance relation has been used. Eqs. (A9) and (A10) (note that $E_{C}-E_{V}=E_{C}^{0}-E_{V}^{0}$ ) are precisely Eqs. (13) and (12) used in Section III.

${ }^{1}$ S. M. Sze, Physics of Semiconductor Devices, 2nd ed. (Wiley, New York, 1981).

${ }^{2}$ E. H. Rhoderick and R. H. Williams, Metal-Semiconductor Contacts, 2nd ed. (OSP, New York, 1987).

${ }^{3}$ H. K. Henisch, Semiconductor Contacts (OUP, Oxford, 1985).

${ }^{4}$ J. Bardeen, Phys. Rev. 71, 717 (1947).

${ }^{5}$ H. A. Bethe, MIT Radiat. Lab. Rep., 43-12 (1942).

${ }^{6}$ W. Schottky, Naturwissenschaften 26, 843 (1938).

${ }^{7}$ C. R. Crowell and S. M. Sze, Solid State Electron. 8, 979 (1965).

${ }^{8}$ H. T. Tseng and C. Y. Wu, Solid State Electron. 30, 383 (1987).

${ }^{9}$ Y. S. Lou and C. Y. Wu, IEEE Trans. Electron. Devices 41, 558 (1994).

${ }^{10}$ S. F. Guo, Solid State Electron. 27, 537 (1984).

${ }^{11}$ K. Maeda and I. Umezu, J. Appl. Phys. 68, 2858 (1990).

${ }^{12}$ Zs. J. Horváth, Appl. Phys. Lett. 65, 511 (1994).

${ }^{13}$ Zs. J. Horváth, J. Appl. Phys. 63, 976 (1988).

${ }^{14}$ P. Muret and A. Deneuville, J. Appl. Phys. 53, 6289 (1982).

${ }^{15}$ C. Y. Wu, J. Appl. Phys. 53, 5947 (1982).

${ }^{16}$ G. Gomila, A. Pérez-Madrid, and M. Rubí, Physica A 233, 208 (1996).

${ }^{17}$ W. Shockley and W. T. Read, Phys. Rev. 87, 835 (1952).

${ }^{18}$ R. N. Hall, Phys. Rev. 87, 387 (1952).

${ }^{19}$ H. C. Card and E. H. Roderick, J. Phys. D 4, 1589 (1971).

${ }^{20}$ M. Sağlam, E. Ayyildiz, A. Gümüs, A. Türüt, H. Efeoğlu, and S. Tüzemen, Appl. Phys. A 62, 269 (1996).

${ }^{21}$ M. Depas, R. L. Van Meirhaeghe, W. H. Laflère, and F. Cardon, SolidState Electron. 37, 433 (1994).

${ }^{22}$ G. Eftekhari, J. Vac. Sci. Technol. B 13, 390 (1995).

${ }^{23}$ K. J. B. M. Nieuwesteeg, M. van der Veen, and T. J. Vink, J. Appl. Phys. 74, 2572 (1993).

${ }^{24}$ K. M. van Vliet, Phys. Rev. 110, 50 (1958). 